\title{
Short communication: Is consumption of a cheese rich in angiotensin-converting enzyme-inhibiting peptides, such as the Norwegian cheese Gamalost, associated with reduced blood pressure?
}

\author{
R. Nilsen, ${ }^{\star 1}$ A. H. Pripp,† A. T. Høstmark, $\ddagger$ A. Haug,§ and S. Skeie* \\ *Department of Chemistry, Biotechnology and Food Science, Norwegian University of Life Sciences, PO Box 5003, N-1432 Ås, Norway \\ †Department of Biostatistics, Epidemiology and Health Economics, Oslo University Hospital, N-0450 Oslo, Norway \\ †Institute of Health and Society, University of Oslo, N-0450 Oslo, Norway \\ $\S$ Department of Animal and Aquacultural Sciences, Norwegian University of Life Sciences, N-1432 Ås, Norway
}

\begin{abstract}
Epidemiological and clinical studies have shown that angiotensin-converting enzyme (ACE)-inhibiting peptides derived from dairy products may decrease blood pressure. These peptides have been identified in many cheeses, and Gamalost, a traditional Norwegian cheese, is particularly rich in these peptides. The aim of this cross-sectional study was to examine whether frequency of Gamalost intake was associated with blood pressure in a Norwegian population sample. Blood pressure and other clinical measurements, including the factors of metabolic syndrome, were obtained from 168 participants $(56 \%$ female, mean age $=51 \mathrm{yr})$ who completed a questionnaire about dietary habits and other healthrelated factors. Mean Gamalost intake was 2 servings per week. The prevalence of hypertension was $23.8 \%$ in the population, with mean systolic and diastolic blood pressures of 128 and $78 \mathrm{mmHg}$, respectively. Intake of Gamalost was inversely associated with systolic blood pressure. Each increase in frequency unit of Gamalost intake corresponded to a reduction in systolic blood pressure of $0.72 \mathrm{mmHg}$, after controlling for sex, age, education, waist circumference, physical activity, smoking status, and dairy food intake. Results from this study indicate that consumption of Gamalost (or other foods rich in ACE-inhibiting peptides) may reduce blood pressure.
\end{abstract}

Key words: cheese, angiotensin-converting enzyme (ACE)-inhibiting peptide, blood pressure, dairy product

\section{Short Communication}

Milk proteins are considered one of the most important sources of bioactive peptides (Korhonen and Pihlanto, 2006) and studies have found that different

Received September 12, 2013.

Accepted January 18, 2014.

${ }^{1}$ Corresponding author: rita.nilsen@nmbu.no cheeses contain several bioactive peptides in varying amounts. Angiotensin-converting enzyme (ACE) is an important enzyme in the renin-angiotensin system, which is one of the pathways that control blood pressure. The effect of ACE is to activate angiotensin II, a vasoconstrictor, and inactivate bradykinin, a vasodilator (Silva and Malcata, 2005; FitzGerald et al., 2004), resulting in an increase in blood pressure. Peptides with ACE-inhibiting or blood pressure (BP)-lowering activity have been identified in many cheeses (Sieber et al., 2010). Cheese and other dairy products are significant sources of saturated fat in the typical western diet (Sonestedt et al., 2011), a fat that may increase the amount of low density lipoprotein (LDL) cholesterol in the blood, which is a risk factor for cardiovascular disease (CVD). However, some studies have found that a higher intake of dairy products is associated with a reduced risk of CVD, and it was recently found that cheese intake is negatively associated with the metabolic syndrome (Høstmark and Tomten, 2011). Part of the reason why cheese may be protective against CVD could be the presence of bioactive peptides.

Gamalost is an autochthonous Norwegian cheese that is naturally low in fat $(<1 \%)$, does not contain salt, and is high in protein (50\%). Details on the production and ripening of Gamalost have been described elsewhere (Qureshi et al., 2012). The cheese was found to have a higher ACE-inhibitory potential than Norvegia, a Gouda-type cheese (Pripp et al., 2006; Qureshi et al., 2012, 2013), and it is one of the cheeses with the highest ACE-inhibitory potential (Sieber et al., 2010). Even though ACE-inhibiting peptides have been found in many cheeses, few studies describe their effect in humans.

The Global Burden of Disease Study 2010 identified high $\mathrm{BP}$ as the leading risk factor for global disease burden (Lim et al., 2012). Hypertension is a major risk factor for $\mathrm{CVD}$, and systolic $\mathrm{BP}>130 \mathrm{mmHg}$ or diastolic $\mathrm{BP}>85 \mathrm{mmHg}$ are 2 of the diagnostic criteria for metabolic syndrome. Cardiovascular disease is the 
most common cause of death in Norway, accounting for about $35 \%$ of all deaths (Folkehelseinstituttet, 2010). The prevalence of hypertension in the adult population in the United States is about 30\% (Yoon et al., 2010), and it has been estimated that a decrease in diastolic $\mathrm{BP}$ of just $5 \mathrm{mmHg}$ can reduce the risk of CVD by $16 \%$ (FitzGerald et al., 2004). Pharmacological treatment of hypertension is often associated with undesirable side effects such as reduced kidney function and hypotension (Haque and Chand, 2008). Consequently, food-derived ACE-inhibitors would be of great interest, as these are not associated with side effects. A meta-analysis of randomized controlled trials on the effect of food-derived peptides on BP found a significant reduction in both systolic and diastolic BP, indicating a possible role for food in the management of mild hypertension (Pripp, 2008). We are not aware of any published observational studies with clinical tests regarding the association between cheese intake and BP. The aim of this epidemiological study was to assess whether the frequency of Gamalost intake was associated with blood pressure and other factors of the metabolic syndrome, in the population of Vik i Sogn, a small community on the Norwegian west coast.

This cross-sectional study was conducted in Vik, Norway, in May 2012. The adult population of Vik comprised the study sample. Participants were recruited through the 4 largest work places in Vik, and one person worked specifically to reach the elderly population. Furthermore, a short article was published in the local newspaper inviting people to participate in the study. One hundred eighty-six people completed the questionnaire. Of those, 5 did not show up for clinical assessment. Pregnant women and participants lacking information on cheese and dairy intake were excluded from the analyses, resulting in a final study sample of 168. Subjects who lacked information on the factors included in the ANOVA or who reported taking BPlowering medications were further excluded from this analysis, resulting in a sample size of 153 . This study was conducted according to the guidelines in the Declaration of Helsinki, and all procedures involving human subjects were approved by Regional Committees for Medical and Health Research Ethics (Oslo, Norway) on April 24, 2012. Written informed consent was obtained from all subjects. The participants were offered breakfast and one Gamalost cheese as compensation for participation.

A questionnaire was developed specifically for this study, based on the previously used food frequency questionnaire from the Oslo Health Study (Mostøl, 2004). In addition to questions about health and physical activity, the questionnaire included a short section on dietary habits, emphasizing dairy intake. Four ques- tions inquired about the intake of cheese, including all cheese, regular (mostly Gouda type) cheeses, brown whey cheese, and Gamalost. Five questions inquired about the intake of other dairy products; for example, milk and yogurt. The variables concerning cheese intake were categorized into rarely or never, 1 to 3 times per month, 1 to 3 times per week, 4 to 6 times per week, 1 to 2 times per day, and 3 times or more per day. For statistical analyses, the midpoint in each category was recalculated into frequency in times per week; that is, $0,0.5,2.0,5.0,10.5$, and 21.0 servings per week, respectively. Total dairy product intake was calculated by summarizing the frequency of intake of all cheese, all milk, and fermented milk.

Height was measured to the nearest $0.1 \mathrm{~cm}$ using a portable stadiometer (Seca 217, Seca, Hamburg, Germany). Weight was measured to the nearest 0.1 $\mathrm{kg}$ using digital scales (TBF-300A Body Composition Analyzer, Tanita, Tokyo, Japan). Waist circumference was measured in accordance with World Health Organization recommendations, at the midpoint between the iliac crest and the lowest rib margin, to the nearest $0.1 \mathrm{~cm}$ (WHO, 2011) using a measuring tape (Seca 201 Circumference measuring tape, Seca). Body mass index (BMI) was calculated as weight $(\mathrm{kg})$ divided by the square of height $(\mathrm{m})$.

Blood pressure was measured according to recommendations from the American Heart Association (Pickering et al., 2005). Participants rested for approximately 10 min before $\mathrm{BP}$ was measured using a Microlife BP A200 BP meter (Microlife, Widnau, Switzerland). Three consecutive measurements were taken, and the average of the second and third measurements was used for analysis (automatically calculated by the blood pressure device). In some cases, the device used 4 measurements to get a more accurate reading. Venous blood samples were drawn in the morning after an overnight fast (approximately 10-12 h), using the Vacutainer system (Becton Dickinson Co., Franklin Lakes, NJ). The samples were centrifuged at $833 \times g$ for 10 min at room temperature, and the serum was separated 1 to $2 \mathrm{~h}$ after the blood was drawn. The serum was frozen to $-20^{\circ} \mathrm{C}$ within $5 \mathrm{~h}$. Fürst Medical Laboratories (Oslo, Norway) conducted the lipid analyses. The measured biochemical markers were total cholesterol, high density lipoprotein (HDL) cholesterol, LDL cholesterol, and triglycerides. Blood glucose was measured in capillary blood by the finger stick method.

Daily physical activity was assessed by 2 questions in the questionnaire; one question regarding amount of leisure time physical activity and one regarding type of physical activity. For statistical analyses, participants were classified into 3 groups of physical activity: sedentary, light physical activity, and moderate to hard 
physical activity. Use of tobacco products was selfreported, and classified into currently using, previously used, and never used.

The SPSS 19.0 software package (IBM Corp., Armonk, NY) was used for the statistical analyses. When appropriate, one-way ANOVA was used to evaluate significance of differences of mean values between the groups of Gamalost intake frequency, using Bonferroni correction for multiple comparisons (Table 1). The statistical significance level was set at $P<0.05$. The independent samples $t$-test was used to assess the difference in means between men and women. Age in years was used as a continuous variable in most analyses, except when regarding the frequency of Gamalost intake (Figure 1), where the participants were grouped into 5 age groups: 18 to $30 \mathrm{yr}, 31$ to $45 \mathrm{yr}, 46$ to $60 \mathrm{yr}, 61$ to $75 \mathrm{yr}$, and $76+$ yr. This was done because of the study sample size and the wide range of ages (61 yr). General linear models were used to assess the association between intake frequency of Gamalost and selected outcomes (systolic and diastolic BP, blood glucose, serum triglycerides, and serum cholesterol), which included adjustment for several potential confounders. These potential confounders were chosen because they are likely to have an effect on BP and could be associated with intake of Gamalost. Several of them are important factors in the treatment and control of hypertension (Krousel-Wood et al., 2004). The adjusted model included 8 factors: sex, age in years, education (total years in school), waist circumference $(\mathrm{cm})$, physical activity (4 levels: sedentary, light physical activity, moderately hard

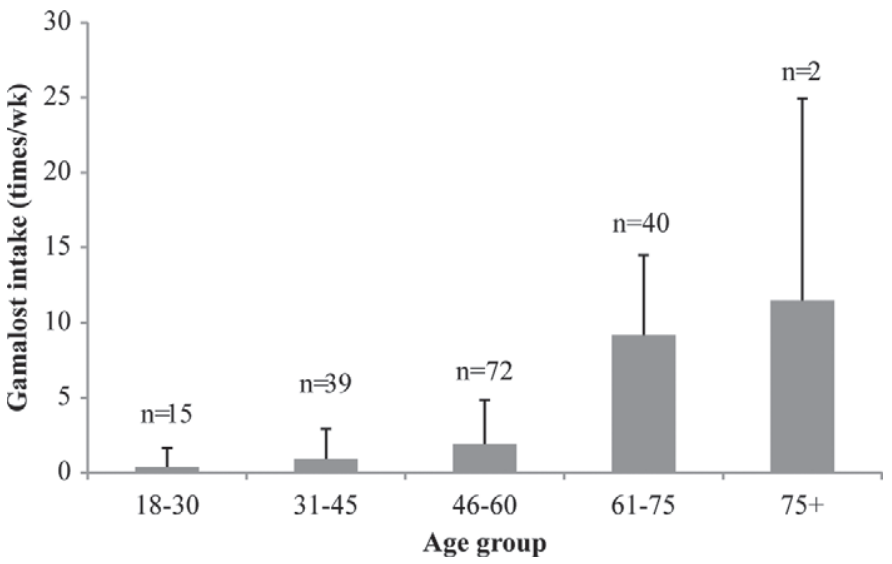

Figure 1. Frequency of Gamalost intake related to the age groups of participants. Mean weekly intakes (times/wk) of Gamalost ( \pm SD) were $0.367(1.29), 0.923$ (1.99), 1.924 (2.95), 9.175 (5.31), and 11.5 (13.44), respectively, for age groups 1 through 5 . Due to the large standard deviations, only the upper standard deviation is shown in this figure.

physical activity, hard physical activity several times per week), smoking (currently, previously, never) and total dairy product intake (6 intake levels, as described above).

Some characteristics of the study sample are shown in Table 1, according to intake frequency of Gamalost (times/wk). Sex, age, waist circumference, education, intake of all cheese, intake of regular cheese, and total dairy product intake were all significantly different between the 6 levels of Gamalost intake frequency. Both age and waist circumference were highest for the 3

Table 1. Some sample characteristics [mean (SD) or \%] by frequency of Gamalost intake

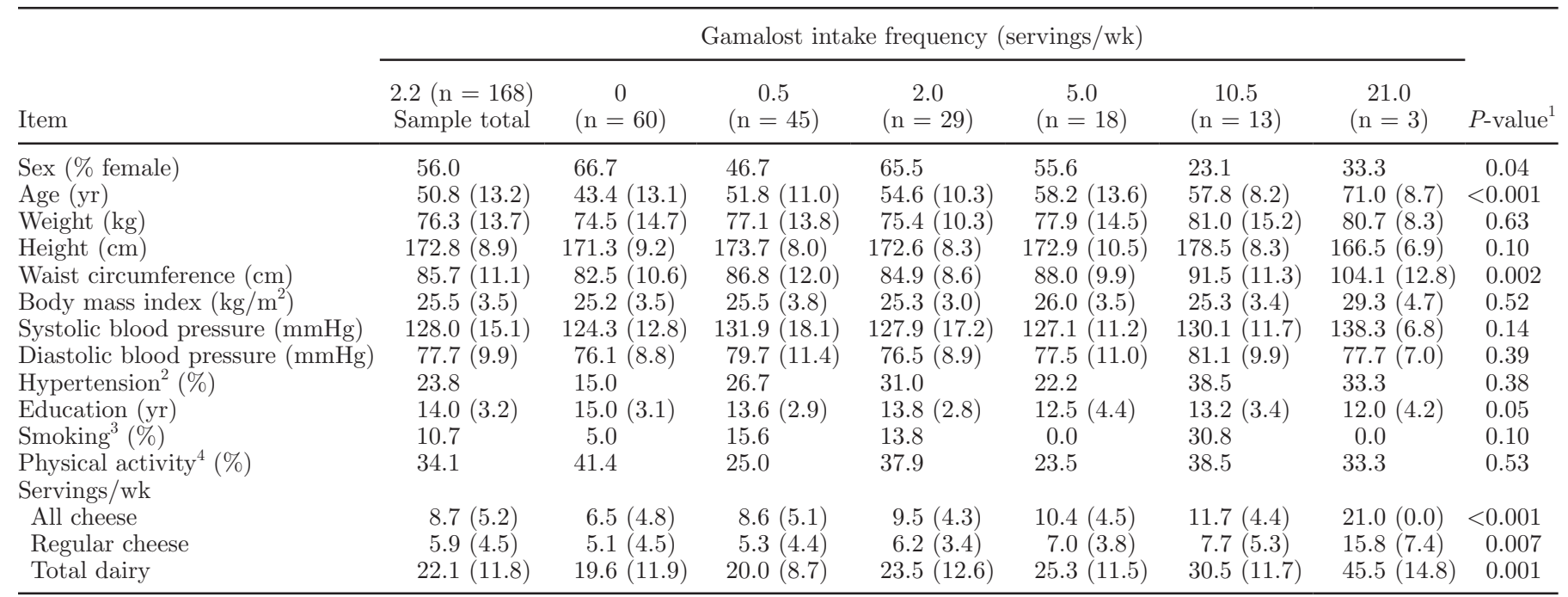

${ }^{1}$ Difference in characteristics between groups (one-way ANOVA test); $P<0.05$.

${ }^{2}$ Percentage who have systolic blood pressure $>140 \mathrm{mmHg}$, diastolic blood pressure $>90 \mathrm{mmHg}$, or both.

${ }^{3}$ Percentage of daily smokers.

${ }^{4}$ Percentage who reported moderate to hard physical activity daily. 
highest intake levels of Gamalost. The mean intake frequency of Gamalost ranged from 0.37 to 11.5 times/wk in the youngest and oldest age groups, respectively. Intake of Gamalost and age showed a significant positive correlation, $\mathrm{r}=0.37(P<0.001,2$-tailed $)$, as expected from Norwegian tradition. For illustration, the mean intake frequency of Gamalost in different age groups is shown in Figure 1. Mean intake of Gamalost was significantly higher in men (mean $=2.96$ servings $/$ wk; $\mathrm{SD}=4.66)$ than in women $($ mean $=1.61 ; \mathrm{SD}=3.04)$, $P=0.03$ (2-tailed; $t$-test). The intakes of other cheeses and total dairy products closely followed the intakes of Gamalost, with the highest intakes being in the highest intake levels of Gamalost. The prevalence of hypertension in the total population was $23.8 \%$ (crude estimate) and was not significantly different between the 6 levels of Gamalost intake.

\section{Gamalost Intake and Blood Pressure}

Cross-sectional associations between 8 selected factors and systolic BP are presented in Table 2 . The crude model shows that only sex, age, and waist circumference were associated with systolic BP. An adjusted model was made to control for potential confounders. The multivariable adjusted model showed a statistically significant inverse association between frequency of intake of Gamalost, and systolic BP [unstandardized regression coefficient used in the general linear model $(\mathbf{B})=-0.72, P=0.03]$. The results show that for each increase in the intake frequency unit of Gamalost (times/wk), systolic BP decreased by $0.72 \mathrm{mmHg}$. Despite the smaller sample size, this is comparable to what has been found in larger population-based studies on cheese consumption (Høstmark and Tomten, 2011; Sonestedt et al., 2011). The prevalence of hypertension, defined as having a systolic BP $>140$ or diastolic BP $>90$ (Chobanian et al., 2003), varied greatly between the subjects in each of the intake levels of Gamalost. The lowest prevalence of hypertension was found in the group that did not consume Gamalost. However, we also observed a significant difference in age between the participants in the 6 levels of Gamalost intake, with the nonconsumers also being the youngest group. It is well established that $\mathrm{BP}$ increases with age (Whelton et al., 2002; Wolf-Maier et al., 2003), which can partly explain why BP was, in the crude univariable statistical analyses, associated positively with intake of Gamalost and, thus, the need to use multivariable statistical models to adjust for possible confounders. The adjusted model also showed that sex (being male), age, and waist circumference were still significantly associated with systolic BP. As shown in Table 3, a nonsignificant inverse association was found between
Gamalost intake and diastolic $\mathrm{BP}(\mathrm{B}=-0.322, P=$ $0.16)$. As with systolic BP, age, sex, and waist circumference were significantly associated with diastolic $\mathrm{BP}$ in the crude model. In the adjusted model, only waist circumference was significantly associated with diastolic BP. This is in accordance with a similar but larger study, which found that a higher frequency of cheese intake was borderline significantly associated with a reduction in systolic BP [standardized regression coefficient $\left.(\beta)=-0.33, P_{\text {trend }}=0.06\right]$ but not with diastolic BP (Sonestedt et al., 2011). Education, physical activity, smoking, or total dairy intake had no effect on either systolic BP or diastolic BP. Although diastolic $\mathrm{BP}$ does not increase after the age of 50 , systolic BP continues to increase throughout life (Chobanian et al., 2003), making management of systolic hypertension very important in the older population. Estimations show that only a $2 \mathrm{mmHg}$ decrease in systolic $\mathrm{BP}$ in a population would reduce mortality from stroke by $6 \%$, whereas a $5 \mathrm{mmHg}$ reduction would reduce mortality from stroke by as much as 14\% (Whelton et al., 2002). If it is confirmed that an increased intake of Gamalost is associated with reduced systolic BP, it is important to establish whether the association is causal and of clinical significance. Provided that the observed association is causal, it would appear that increased intake of a cheese with a high ACE inhibitory activity, such as Gamalost, to the levels seen in the highest intake frequency group in this population might reduce BP, and thus mortality, in a population. Although some studies on the effect of milk-derived bioactive peptides on hypertension have shown an effect in normotensive subjects, most successful intervention trials have been on subjects with mild or moderate hypertension (Engberink et al., 2008). It is tempting to speculate that we would find a larger effect of Gamalost on BP if more participants in the study had hypertension.

In addition to BP, other factors of the metabolic syndrome were included in this study. Table 4 shows the significant associations in the adjusted model for these factors. Nonsignificant associations were excluded from this table. As can be seen from the table, increased waist circumference and smoking were both positively associated with increased blood glucose and triglycerides. Furthermore, serum HDL level was significantly negatively associated with being male $(\mathrm{B}=-0.244, P$ $=0.001)$ and inversely associated with waist circumference $(\mathrm{B}=-0.014, P<0.001)$. With increasing intake frequency of Gamalost, a significant increase in total serum cholesterol $(\mathrm{B}=0.068, P=0.01)$ occurred, which was also reflected in LDL cholesterol $(\mathrm{B}=0.061$, $P=0.03)$ but not HDL cholesterol.

Results on the role of cheese in the etiology of different CVD have been contradictory. Most studies on 
Table 2. Crude and adjusted associations between 8 selected factors and systolic blood pressure $(\mathrm{n}=153)$

\begin{tabular}{|c|c|c|c|c|c|c|}
\hline Item & \multicolumn{3}{|c|}{ Crude } & \multicolumn{3}{|c|}{ Adjusted } \\
\hline Gamalost intake $^{2}$ & 0.179 & 0.61 & $-0.513,0.872$ & -0.720 & $0.03^{*}$ & $-1.380,-0.059$ \\
\hline Age & 0.397 & $<0.001^{*}$ & $0.232,0.562$ & 0.311 & $0.001 *$ & $0.130,0.492$ \\
\hline Years of education & -0.685 & 0.07 & $-1.415,0.045$ & -0.169 & 0.63 & $-0.863,0.525$ \\
\hline Waist circumference & 0.674 & $<0.001^{*}$ & $0.485,0.864$ & 0.436 & $<0.001^{*}$ & $0.194,0.678$ \\
\hline
\end{tabular}

${ }^{1} \mathrm{~B}$ represents the mean change in blood pressure.

${ }^{2}$ Frequency of intake: 0, 0.5, 2.0, 5.0, 10.5 and 21.0 times/wk.

$* P<0.05$.

this subject are on the role of dairy products, with cheese comprising only a small subsection of the study. Furthermore, the CVD umbrella can be very unspecific, with the outcome measures being very different from study to study. Moreover, the nutritional composition of cheese is highly variable, making it difficult to generalize results. A recent meta-analysis of prospective cohort studies on the role of dairy in hypertension found that per $200 \mathrm{~g} / \mathrm{d}$ of total dairy, the risk of hypertension was lowered by $3 \%$ (risk ratio: $0.97,95 \%$ CI: $0.95,0.99$; Soedamah-Muthu et al., 2012). However, the same meta-analysis of 51,007 individuals with mean cheese intakes ranging from 10 to $43 \mathrm{~g} / \mathrm{d}$ showed no effect of cheese intake on hypertension (risk ratio $=1.00,95 \%$ CI: $0.98,1.03)$. This might, however, reflect the large variation in the amount and activity of bioactive peptides in different cheeses and dairy products.

Diets aimed at reducing hypertension, such as the DASH diet (Dietary Approaches to Stop Hypertension; Sacks et al., 2001), often emphasize a high intake of lowfat dairy products. Gamalost contains only negligible amounts of fat $(<1 \mathrm{~g} / 100 \mathrm{~g})$, making it suitable in these diets. The role of salt $(\mathrm{NaCl})$ in the development of hy- pertension is much debated, but it is generally accepted that limiting salt intake is favorable in preventing hypertension. Salt is usually an important ingredient in the cheese-making process, contributing both to a desirable flavor and texture profile of the cheese and to microbial safety (Guinee and Fox, 2004). This, together with the high fat content of most cheeses, is the reason why cheese is generally not recommended in heart-healthy diets. Gamalost, however, is made without the addition of salt. Thus, if consumers eat Gamalost instead of other cheeses (rather than in addition to other cheeses), it could be argued that the concomitant reduction in fat and salt intake is the cause of the reduction in BP. However, as can be seen in Table 1, it seems that the most frequent consumers of Gamalost also have the highest intakes of all cheese and regular cheese. It is not possible from our study to assess if the found association between systolic BP and intake of Gamalost is due specifically to the high ACE-inhibitory potential of Gamalost, as the participants who consumed Gamalost most frequently also consumed more of other cheeses. Thus, they also consumed a higher amount of other nutrients found in cheese, such as calcium and potassium.

Table 3. Crude and adjusted associations between 8 selected factors and diastolic blood pressure $(\mathrm{n}=153)$

\begin{tabular}{|c|c|c|c|c|c|c|}
\hline Item & \multicolumn{3}{|c|}{ Crude } & \multicolumn{3}{|c|}{ Adjusted } \\
\hline Gamalost intake $^{2}$ & 0.095 & 0.68 & $-0.361,0.551$ & -0.322 & 0.16 & $-0.776,0.131$ \\
\hline Age & 0.172 & $0.009^{*}$ & $0.043,0.301$ & 0.093 & 0.14 & $-0.031,0.218$ \\
\hline Years of education & -0.273 & 0.33 & $-0.830,0.284$ & 0.059 & 0.81 & $-0.417,0.536$ \\
\hline Waist circumference & 0.432 & $<0.001^{*}$ & $0.299,0.564$ & 0.393 & $<0.001^{*}$ & $0.227,0.558$ \\
\hline
\end{tabular}

${ }^{1} \mathrm{~B}$ represents the mean change in blood pressure.

${ }^{2}$ Frequency of intake: $0,0.5,2.0,5.0,10.5$, and 21.0 times/wk.

${ }^{*} P<0.05$. 
Table 4. Adjusted associations between selected factors and blood glucose, triglycerides, and cholesterol $(\mathrm{n}=153)^{1}$

\begin{tabular}{|c|c|c|c|c|c|c|c|c|c|c|}
\hline \multirow[b]{2}{*}{ Item } & \multicolumn{2}{|c|}{ Blood glucose } & \multicolumn{2}{|c|}{ Cholesterol } & \multicolumn{2}{|c|}{ Triglycerides } & \multicolumn{2}{|c|}{$\begin{array}{c}\text { High-density } \\
\text { lipoprotein cholesterol }\end{array}$} & \multicolumn{2}{|c|}{$\begin{array}{l}\text { Low-density } \\
\text { lipoprotein } \\
\text { cholesterol }\end{array}$} \\
\hline & $\mathrm{B}^{2}$ & $P$-value & B & $P$-value & B & $P$-value & $\mathrm{B}$ & $P$-value & B & $P$-value \\
\hline Gamalost intake $^{3}$ & -0.040 & 0.10 & 0.068 & 0.01 & 0.010 & 0.47 & 0.011 & 0.24 & 0.061 & 0.03 \\
\hline Sex, if male & 0.106 & 0.57 & -0.195 & 0.32 & 0.005 & 0.96 & -0.244 & 0.001 & 0.102 & 0.62 \\
\hline Waist circumference & 0.026 & 0.004 & 0.006 & 0.50 & 0.025 & $<0.001$ & -0.014 & $<0.001$ & 0.017 & 0.08 \\
\hline
\end{tabular}

${ }^{1}$ Only significant factors are shown; other factors were analyzed but were not significant.

${ }^{2} \mathrm{~B}$ represents the mean change in blood pressure.

${ }^{3}$ Frequency of intake: 0, 0.5, 2.0, 5.0, 10.5 and 21.0 times/wk.

Both dietary calcium and potassium have been shown to lower systolic and diastolic BP (Griffith et al., 1999; Houston, 2011) and to reduce the risk of hypertension in, for example, middle-aged and older women (Wang et al., 2008). In a study with hypertensive subjects, Calpis, a fermented milk that, like Gamalost, has a naturally high ACE-inhibitory potential, was compared with a placebo drink with equal calcium and potassium contents (Hata et al., 1996). In that study, a significant decrease in both systolic and diastolic BP was observed with Calpis only, suggesting that the effect was independent of the calcium and potassium contents.

The main strength of the present study is that a cheese shown to have a high ACE-inhibitory activity was asked about specifically in a food frequency questionnaire, not just as part of a question about total cheese or total dairy. The study sample was relatively homogeneous, in terms of ethnicity, place of birth, and environmental factors. Furthermore, all BP measurements were done under the same conditions - in the morning after an overnight fast. There was no interobserver error in the anthropometric measurements, because the same observer did all measurements. Even though the sample size was limited, we were able to recruit just under $10 \%$ of the eligible population in Vik i Sogn (total population of 2,768 in 2010). One limitation is the nature of the study design itself; namely, that it is a small cross-sectional study. The design cannot provide any evidence for a true cause-and-effect association, but the results may serve to suggest a direction for future research. To clarify whether intake of Gamalost does prevent hypertension and might reduce $\mathrm{BP}$ in hypertensive subjects, a large randomized intervention study would be needed. Therefore, an intervention trial comparing a possible BP-reducing influence of Gamalost with other types of cheese is currently in progress.

In summary, intake of Gamalost, a cheese with a high concentration of ACE-inhibiting peptides, was negatively associated with systolic BP in a Norwegian population with a high prevalence of Gamalost users.
The association remained after controlling for sex, age, education, waist circumference, physical activity, smoking habits, and total intake of dairy products. Total dairy intake or total cheese intake was not associated with blood pressure in this study.

\section{ACKNOWLEDGMENTS}

The authors acknowledge the Norwegian Research Council (Oslo), the Norwegian Foundation for Research Levy on Agricultural Products (Oslo), the Norwegian Agricultural Agreement Research Fund, and TINE SA (Oslo, Norway) for financial support. We also thank our contacts in Vik who very kindly helped us recruit participants for this study. TINE Meieriet Vik (Vik i Sogn, Norway) is thanked for their participation, especially for providing breakfast for the participants and lending us their office space. Finally, Eirin Huseby, master student at the Norwegian University of Life Sciences, and Erna Skeie, a local nurse in Vik, Norway, are very much appreciated for all their help in Vik.

\section{REFERENCES}

Chobanian, A. V., G. L. Bakris, H. R. Black, W. C. Cushman, L. A. Green, J. L. Izzo, D. W. Jones, B. J. Materson, S. Oparil, J. T. Wright, and E. J. Roccella. 2003. Seventh report of the Joint National Committee on Prevention, Detection, Evaluation, and Treatment of High Blood Pressure. Hypertension 42:1206-1252.

Engberink, M. F., E. G. Schouten, F. J. Kok, L. A. J. van Mierlo, I. A. Brouwer, and J. M. Geleijnse. 2008. Lactotripeptides show no effect on human blood pressure: Results from a double-blind randomized controlled trial. Hypertension 51:399-405. http:// dx.doi.org/10.1161/hypertensionaha.107.098988.

FitzGerald, R. J., B. A. Murray, and D. J. Walsh. 2004. Hypotensive peptides from milk proteins. J. Nutr. 134(Suppl. 4):980S-988S.

Folkehelseinstituttet. 2010. Folkehelserapport 2010 Helsetilstanden i Norge (Public Health Report 2010 Health in Norway). Folkehelseinstituttet (Norwegian Institute of Public Health), Oslo, Norway.

Griffith, L. E., G. H. Guyatt, R. J. Cook, H. C. Bucher, and D. J. Cook. 1999. The influence of dietary and nondietary calcium supplementation on blood pressure: An updated meta-analysis of randomized controlled trials. Am. J. Hypertens. 12:84-92.

Guinee, T. P., and P. F. Fox. 2004. Salt in cheese: Physical, chemical and biological aspects. Pages 207-259 in Cheese-Chemistry, 
Physics and Microbiology. Volume 1: General Aspects. 3rd ed. P. F. Fox, P. L. H. McSweeney, T. M. Cogan, and T. P. Guinee, ed. Elsevier Academic Press, London, UK.

Haque, E., and R. Chand. 2008. Antihypertensive and antimicrobial bioactive peptides from milk proteins. Eur. Food Res. Technol. 227:7-15. http://dx.doi.org/10.1007/s00217-007-0689-6.

Hata, Y., M. Yamamoto, M. Ohni, K. Nakajima, Y. Nakamura, and T. Takano. 1996. Placebo-controlled study of the effect of sour milk on blood pressure in hypertensive subjects. Am. J. Clin. Nutr. 64:767-771.

Høstmark, A. T., and S. E. Tomten. 2011. The Oslo health study: Cheese intake was negatively associated with the metabolic syndrome. J. Am. Coll. Nutr. 30:182-190.

Houston, M. C. 2011. The importance of potassium in managing hypertension. Curr. Hypertens. Rep. 13:309-317. http://dx.doi. org/10.1007/s11906-011-0197-8.

Korhonen, H., and A. Pihlanto. 2006. Bioactive peptides: Production and functionality. Int. Dairy J. 16:945-960.

Krousel-Wood, M. A., P. Muntner, J. He, and P. K. Whelton. 2004. Primary prevention of essential hypertension. Med. Clin. North Am. 88:223-238.

Lim, S. S., T. Vos, A. D. Flaxman, G. Danaei, K. Shibuya, and H. Adair-Rohani et al. 2012. A comparative risk assessment of burden of disease and injury attributable to 67 risk factors and risk factor clusters in 21 regions, 1990-2010: A systematic analysis for the Global Burden of Disease Study 2010. Lancet 380:2224-2260. http://dx.doi.org/10.1016/S0140-6736(12)61766-8.

Most $\varnothing l$, A. 2004. Dietary assessment-The weakest link? A dissertation exploring the limitations to questionnaire based methods of dietary assessment. $\mathrm{PhD}$ Thesis. University of Oslo, Oslo, Norway

Pickering, T. G., J. E. Hall, L. J. Appel, B. E. Falkner, J. Graves, M. N. Hill, D. W. Jones, T. Kurtz, S. G. Sheps, and E. J. Roccella. 2005. Recommendations for blood pressure measurement in humans and experimental animals. Hypertension 45:142-161. http://dx.doi.org/10.1161/01.HYP.0000150859.47929.8e.

Pripp, A. H. 2008. Effect of peptides derived from food proteins on blood pressure: A meta-analysis of randomized controlled trials. Food Nutr. Res. 52: http://dx.doi.org/10.3402/fnr.v52i0.1641.

Pripp, A. H., R. Sorensen, L. Stepamak, and T. Sorhaug. 2006. Relationship between proteolysis and angiotensin-I-converting enzyme inhibition in different cheeses. Lebenson. Wiss. Technol. 39:677683. http://dx.doi.org/10.1016/j.lwt.2005.03.018.

Qureshi, T. M., G. E. Vegarud, R. K. Abrahamsen, and S. Skeie. 2012. Characterization of the Norwegian autochthonous cheese Gamalost and its angiotensin I-converting enzyme (ACE) inhibitory activity during ripening. Dairy Sci. Technol. 92:613-625. http://dx.doi.org/10.1007/s13594-012-0078-1.

Qureshi, T. M., G. E. Vegarud, R. K. Abrahamsen, and S. Skeie. 2013. Angiotensin 1-converting enzyme (ACE) inhibitory activity of the
Norwegian autochthonous cheese Gamalost and Norvegia after in vitro human gastrointestinal digestion. J. Dairy Sci. 96:838-853. http://dx.doi.org/10.3168/jds.2012-5993.

Sacks, F. M., L. P. Svetkey, W. M. Vollmer, L. J. Appel, G. A. Bray, D. Harsha, E. Obarzanek, P. R. Conlin, E. R. Miller, D. G. Simons-Morton, N. Karanja, and P. H. Lin. for the DASH-Sodium Collaborative Research Group. 2001. Effects on blood pressure of reduced dietary sodium and the Dietary Approaches to Stop Hypertension (DASH) diet. N. Engl. J. Med. 344:3-10. http:// dx.doi.org/10.1056/NEJM200101043440101.

Sieber, R.. U. Butikofer, C. Egger, R. Portman, B. Walther, and D. Wechsler. 2010. ACE-inhibitory activity and ACE-inhibiting peptides in different cheese varieties. Dairy Sci. Technol. 90:47-73. http://dx.doi.org/10.1051/dst/2009049.

Silva, S. V., and F. X. Malcata. 2005. Caseins as source of bioactive peptides. Int. Dairy J. 15:1-15. http://dx.doi.org/10.1016/j. idairyj.2004.04.009.

Soedamah-Muthu, S. S., L. D. M. Verberne, E. L. Ding, M. F. Engberink, and J. M. Geleijnse. 2012. Dairy consumption and incidence of hypertension: A dose-response meta-analysis of prospective cohort studies. Hypertension 60:1131-1137. http://dx.doi.org/10.1161/ hypertensionaha.112.195206.

Sonestedt, E., E. Wirfalt, P. Wallstrom, B. Gullberg, M. Orho-Melander, and B. Hedblad. 2011. Dairy products and its association with incidence of cardiovascular disease: The Malmo diet and cancer cohort. Eur. J. Epidemiol. 26:609-618. http://dx.doi org/10.1007/s10654-011-9589-y.

Wang, L., J. E. Manson, J. E. Buring, I. M. Lee, and H. D. Sesso. 2008. Dietary intake of dairy products, calcium, and vitamin D and the risk of hypertension in middle-aged and older women. Hypertension 51:1073-1079. http://dx.doi.org/10.1161/hypertensionaha. 107.107821

Whelton, P., J. He, and L. Appel. 2002. Primary prevention of hypertension: Clinical and public health advisory from the national high blood pressure education program. JAMA 288:1882-1888. http://dx.doi.org/10.1001/jama.288.15.1882.

WHO (World Health Organization). 2011. Waist circumference and waist-hip ratio: Report of a WHO expert consultation. WHO, Geneva, Switzerland.

Wolf-Maier, K., R. S. Cooper, J. R. Banegas, S. Giampaoli, H. W. Hense, M. Joffres, M. Kastarinen, N. Poulter, P. Primatesta, F. Rodríguez-Artalejo, B. Stegmayr, M. Thamm, J. Tuomilehto, D. Vanuzzo, and F. Vescio. 2003. Hypertension prevalence and blood pressure levels in 6 European countries, Canada, and the United States. JAMA 289:2363-2369. http://dx.doi.org/10.1001/ jama.289.18.2363

Yoon, S. S., Y. Ostchega, and T. Louis. 2010. Recent trends in the prevalence of high blood pressure and its treatment and control, 1999-2008. NCHS Data Brief 48:1-8. 